\title{
DISCUSSION
}

\section{Lateral stability of compressible walls}

\author{
T. D. O'ROURKE (1987). Géotechnique 37, No. 2, 145-149
}

\section{Prof. A. O. Uriel, Agromán, Spain}

The Author has shown how the compressibility of a retaining wall may affect its stability. Wall settlements relative to fill can produce a shear reversal at the soil-wall interface which modifies the inclination of the active pressure. The tangential component of the resultant at the wall base increases whereas the normal one decreases. The factor of safety against sliding decreases accordingly.

There may be some other reasons for lateral stability reductions related to underlying soil compressibility and wall stiffness. As an example, the case of large lateral displacements of a harbour quay is briefly described.

Very often gravity walls for quays consist of massive concrete blocks. The vertical joints between the blocks on each horizontal layer commonly alternate with those of the contiguous layers in order to avoid continuous vertical joints. This, together with the in situ concrete superstructure, confers on the wall a relatively large stiffness in the longitudinal direction of the quay. Because of this, if the compressibility of the underlying soil in that direction is not very uniform, an arching effect within the wall can develop in coincidence with the softer ground.

An extension to an existing quay in Cádiz, $220 \mathrm{~m}$ long, was recently built for container operation. The total quay height is $16 \mathrm{~m}$ (at low water springs, the crest is $5 \mathrm{~m}$ above water level, giving $11 \mathrm{~m}$ depth) which is rather large for this kind of gravity wall. The quay was founded on a $2 \mathrm{~m}$ high berm of rockfill and coarse gravel resting on the natural ground. This consists of medium plasticity clayey marls of moderate to firm consistency.

Some time after wall construction, differential settlements of up to about $25 \mathrm{~cm}$ were observed in a $50 \mathrm{~m}$ long portion of the quay crest. When the granular and general backfill were being placed and had reached an elevation of about $5 \mathrm{~m}$ below the crest, relatively sudden lateral displacements took place in the same quay portion. The maximum displacement was similar to the maximum settlement.

The displacement and settlement profiles roughly coincide. According to diver observations, the lateral displacements took place at several of the deepest horizontal joints. The fill placement was stopped and 2 to $3 \mathrm{~m}$ were exacavated in front of the affected quay length. Site investigation records were then re-examined. It was found that the underlying soil stiffness and strength at the settlement zone was clearly less than that elsewhere, but still sufficient for a gravity retaining structure.

Long walls are usually studied as plane or twodimensional problems. Three-dimensional effects are rarely taken into account. The stability is considered adequate if, at each potential slip plane, the product of the shear force and the safety factor is less than the product of the normal force and the assumed friction coefficient.

Wall stability is based on the assumption that the normal force due to wall weight exists at the computed magnitude. But if the underlying soil is not uniform in compressibility and the longitudinal wall stiffness is large enough for an arching effect to develop, this ceases to be the case, as some of the force is transferred to the base of the adjacent wall portions founded on stiffer ground. This was the explanation given in the report on this example by myself. While the wall settlements did not affect the earth pressures and, therefore, the shear force remained unchanged, the normal force had locally decreased.

However, from O'Rourkes' Paper, it is possible that the shear reversal at the wall-fill interface caused by the wall settlements, which increased the shear force and decreased that on the horizontal joints and brought down the safety factor, could have contributed significantly to the observed lateral displacements.

Furthermore, it was found that, at about half of the horizontal joints, a thin plastic sheet was visible between the concrete blocks. This was the film used at the block casting area to prevent the floor slab being glued to the blocks, which had not been detached from some of them. Shear tests carried out in a large shear box with and without the plastic sheet between the concrete samples revealed a friction decrease from $30^{\circ}$ to $10^{\circ}$.

The solution was very costly and consisted of a combination of horizontal steel tendons anchored in the fill at some distance from the wall, and vertical micropiles fastening the concrete blocks and carrying their weight to deeper and stiffer ground layers. 


\section{Author's reply}

The Writer has provided an interesting and informative case history The total quay wall height of $16 \mathrm{~m}$ is relatively large and the weight of the concrete block wall is sufficient to have contributed significantly to the observed wall settlements. The presence of plastic sheets along horizontal block layers would have reduced the lateral shear capacity of the wall. This observation emphasizes the need for quality control during construction, as well as dowelling or otherwise providing for enhanced shear connectivity between contiguous block courses. Installation and post tensioning of vertical steel tendons can provide an effective means of increasing the normal stresses on horizontal layers. This technique has been used successfully during the rehabilitation of concrete-block quay walls elsewhere (Chapman, 1986).

Although the model presented by the Author is based on some simplifying assumptions, it nevertheless should alert designers and contractors to the potentially detrimental effects of wall settlement, especially when gabion, crib, or block walls are constructed at heights exceeding $7 \mathrm{~m}$. Measures to prevent or compensate for wall settlement are often easy to develop and may avert considerable problems at a later stage.

\section{REFERENCE}

Chapman, K. R. (1986). Earth tieback support for an existing flood-wall. Tiebacks for Bulkheads. Geotechnical Special Publication No. 4, pp. 10-32. New York: American Society of Civil Engineers 